\title{
Experimental Measurement of Single-Wall Carbon Nanotube Torsional Properties
}

\author{
A. R. Hall, ${ }^{1}$ L. An, ${ }^{2}$ J. Liu, ${ }^{2}$ L. Vicci, ${ }^{3}$ M. R. Falvo, ${ }^{1}$ R. Superfine,,${ }^{1,3,4}$ and S. Washburn ${ }^{1,3,4,5}$ \\ ${ }^{1}$ Curriculum in Applied and Materials Sciences, University of North Carolina at Chapel Hill, Chapel Hill, North Carolina 27599, USA \\ ${ }^{2}$ Department of Chemistry, Duke University, Durham, North Carolina 27708, USA \\ ${ }^{3}$ Department of Computer Science, University of North Carolina at Chapel Hill, Chapel Hill, North Carolina 27599, USA \\ ${ }^{4}$ Department of Physics and Astronomy, University of North Carolina at Chapel Hill, Chapel Hill, North Carolina 27599, USA \\ ${ }^{5}$ Department of Biomedical Engineering, University of North Carolina at Chapel Hill, Chapel Hill, North Carolina 27599, USA
}

(Received 2 May 2006; published 28 June 2006)

\begin{abstract}
We report on the characterization of nanometer-scale torsional devices based on individual singlewalled carbon nanotubes as the spring elements. The axial shear moduli of the nanotubes are obtained through modeling of device reaction to various amounts of applied electrostatic force and are compared to theoretical values.
\end{abstract}

DOI: 10.1103/PhysRevLett.96.256102

PACS numbers: 68.60.Bs, 81.05.Tp

It has been speculated that the unique material properties of carbon nanotubes (CNT) will make them ideal candidates for use in future nanoelectromechanical systems (NEMS) [1]. Atomically ordered, they demonstrate low chemical reactivity [2], low defect density [3], and high mechanical resilience [4], which make them prime candidates for integration into device architectures requiring repeated actuation with little degradation over time.

One device that has garnered attention recently incorporates the CNT as a torsional spring for a suspended metal platform. This geometry has been used to demonstrate a history-dependent effect in the spring constant of multiwalled nanotubes [5], as well as rotor devices [6,7] and resonant oscillators [8]. More recently, a torsional pendulum was built on a single-walled nanotube (SWNT) [9] and was used to demonstrate the high torsional elasticity of the material. In the latter study, the torsional spring constant of system was assumed solely from theory and device geometry. Here, we present the first measurement of the shear modulus of an individual SWNT.

The device fabrication follows closely the methods reported previously [10], with notable exceptions only in the starting material. For this work, SWNT were grown directly onto degenerately doped silicon wafers with a $1 \mu \mathrm{m}$ thermal oxide layer with chemical vapor deposition [11]. Analysis of the resultant material by atomic force microscopy (AFM) revealed a mean tube diameter of $0.97 \mathrm{~nm}$ [Fig. 1(a)], which indicates that the sample comprises predominantly individual SWNTs. Electron beam lithography and thermal deposition of $10 \mathrm{~nm} \mathrm{Cr}$ and $75 \mathrm{~nm} \mathrm{Au}$ were then used to define two metal anchors $(4 \mu \mathrm{m} \times$ $4 \mu \mathrm{m}$ ) surrounding a smaller platform (typically $300 \mathrm{~nm} \times 250 \mathrm{~nm}$ ) in line along an individual CNT. The separation between the anchors is typically $1 \mu \mathrm{m}$. The center platform acts as the paddle device with typical mass of about $10^{-16} \mathrm{~kg}$ and typical moment of inertia on the order of $10^{-30} \mathrm{~kg} \mathrm{~m}^{2}$. Buffered hydrofluoric acid was used to etch $300-500 \mathrm{~nm}$ of oxide from beneath the device followed by drying with supercritical $\mathrm{CO}_{2}$, leaving the paddle suspended above the substrate and supported only by the SWNT [Fig. 1(b)].

Device actuation was performed in situ in a scanning electron microscope (SEM) by applying a voltage between the device and the backgate electrode. Because of the SWNT axis being asymmetric with the paddle itself, the associated electrostatic attraction forces contribute to a rotation. We note that lateral motion of the device is assumed to be negligible due to the high axial rigidity of SWNTs relative to its twisting rigidity [12]. Application of a low dc bias (typically $2-4 \mathrm{~V}$ ) between the device and the backgate electrode was found to deflect the paddle torsionally to nearly $90^{\circ}$. Figure 2(a) shows a series of images of a SWNT-supported device under deflections of $56^{\circ}, 77^{\circ}$, and $87^{\circ}$ from left to right. All measured devices show a clear increase in deflection angle for higher applied bias.

Similarly to previous work on SWNTs [9], we note an initial actuation of the paddle due to the imaging beam. For our system, this deflection was typically between $30^{\circ}$ and $60^{\circ}$. We also note that prolonged imaging of the device resulted in further deflection, up to $90^{\circ}$. This may be attributable to electron pressure or increased charging of the remnant oxide layer beneath the paddle. For this reason, all experiments were carried out within time frames that did not demonstrate measurable electron beaminduced deflection. It is also assumed that for the duration of the experiments, the torque associated with this deflection is constant.

Upon removal of the bias, each device returned to its initial position. This indicates both the elastic behavior of the SWNT and its secure pinning at the anchors, as well as the minimal influence of electron beam-induced charging on the deflection measurements.

For each device studied, a series of SEM images were taken normal to the substrate at various applied biases. Each successive image of the device was taken at a static position Through direct measurements of these images, 

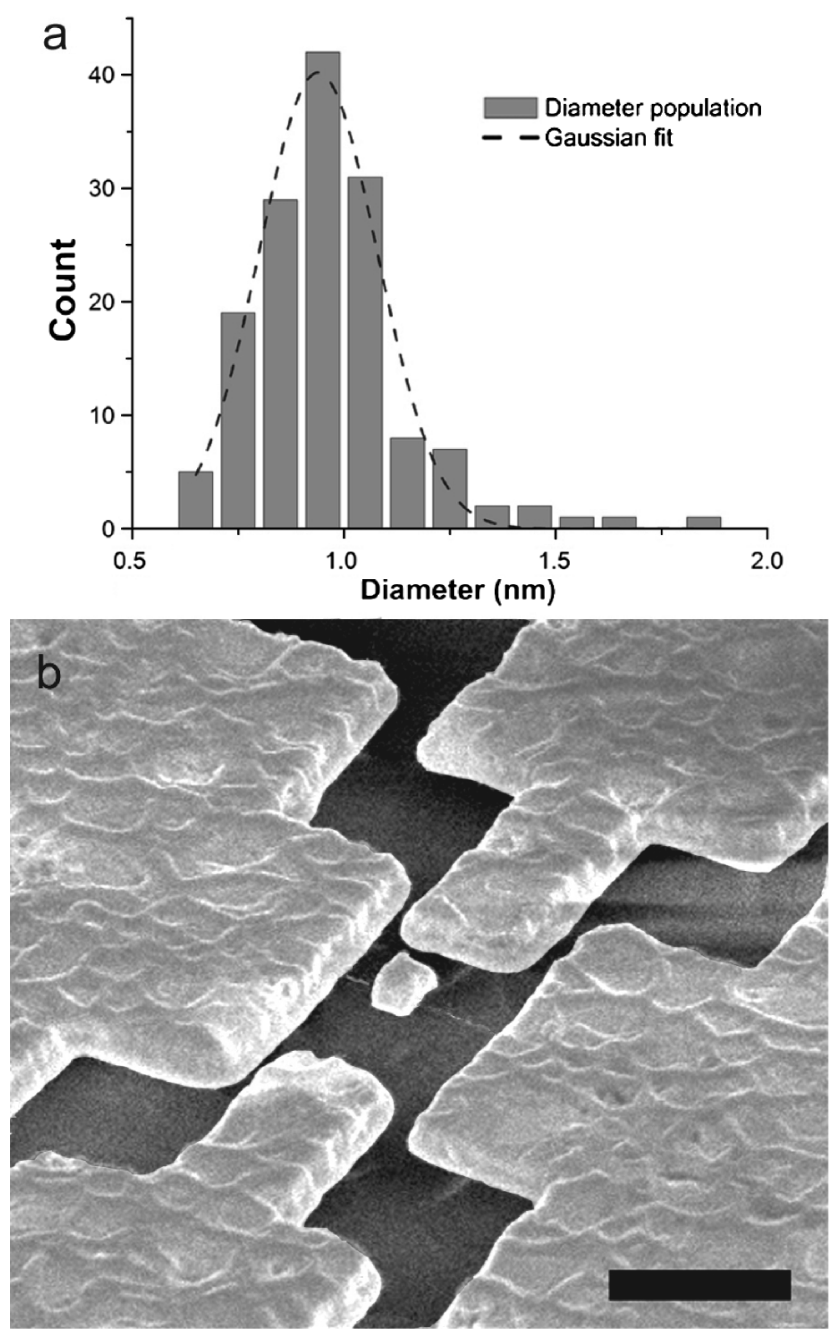

FIG. 1. (a) Diameter distribution of SWNT material measured by AFM and (b) a suspended SWNT device. Scale bar corresponds to $1 \mathrm{~mm}$.

device dimensions including SWNT lengths, paddle length, paddle width, and moment arm were obtained. The degree of deflection was calculated from the projected image length of the paddle.

This information along with AFM analysis of actual etch depth permitted an accurate computer model of each deflection to be built [Fig. 2(b)]. Finite element (FE) analysis of the applied field on the system could then be performed. Examples of the calculated electric potential of a device are shown in Fig. 2. Note that we do not include the SWNT itself in the model as its perturbation of the electric field is assumed to be negligible.

Through this model, surface charge density $(\sigma)$ at each point on the surface of a paddle was obtained, where

$$
\sigma=\varepsilon_{o} E \cdot \hat{n}
$$

Here, $\varepsilon_{o}$ is the permittivity assumed for vacuum, $\hat{n}$ is the local unit vector perpendicular to the paddle surface, and $\vec{E}$ is the electric field calculated with the FE program. The force on an infinitesimal area $d A$ of the paddle surface is

$$
d \vec{F}=\sigma \vec{E} d A
$$

The paddle is a conductor, so the incident electrostatic field $\vec{E}_{\text {surf }}=E \hat{n}$ must be normal to its surface. Accordingly, we have

$$
d \vec{F}=\frac{1}{\varepsilon_{o}} \sigma^{2} \hat{n} d A
$$

The incremental torque on the paddle contributed by $d A$ is $d \vec{T}=\vec{R} \times \overrightarrow{d F}$, where $R$ is the distance of $d A$ from the SWNT axis. Therefore, the total electrostatic torque on the paddle is given by the surface integral

$$
T=\frac{1}{\varepsilon_{o}} \int_{\text {surface }} \sigma^{2} \vec{R} \times \hat{n} d A .
$$

By construction, the model is composed of six individual surfaces [Fig. 3(a)] from which to calculate contributions to the net torque. Four faces of the paddle (top, bottom, and two ends) experience forces that contribute to this net value. Forces on the sides of the paddle (facing the anchors) have no component perpendicular to the axis of rotation and thus do not contribute. Therefore, summing the surface integrals over the four relevant faces [Fig. 3(b)] completes the electrostatic torque calculation.

The net torque varied with the applied bias, but was typically on the order of $10^{-18} \mathrm{~N} \mathrm{~m}$ for the biases in these experiments. This value is related to the shear modulus, $G$, of the SWNT spring through the equation [13]

$$
G=\frac{T\left(l_{1}+l_{2}\right)}{r^{3} t \pi \theta},
$$

where $l_{1}$ and $l_{2}$ are the nanotube length to the left and right of the paddle, respectively, $r$ is the nanotube radius, $\theta$ is the deflection angle, and $t$ is the wall thickness. We use $t=$ $3.4 \AA$ as in Ref. [14].

We found that the SWNT behaves as a linear torsional spring [Fig. 4(a)]. We note that the deflection limit of our setup is $90^{\circ}$ (perpendicular to the substrate) and, as that limit is approached, the measurement may take on added uncertainty. By averaging all measured values for a device, we arrive at a value for $G$ of the particular SWNT. As shown in Fig. 4(b), the inferred values are in agreement with predictions [14]. The average value for the shear moduli of all devices is $G=0.41 \pm 0.36 \mathrm{TPa}$. The inset in Fig. 4(a) shows that there is no detectable nonlinearity in the response, and from theoretical analysis, none is expected for our modest levels of strain [15].

The largest error in our measurement stems from our inability to measure the diameter of each SWNT accurately once the devices are suspended. Our calculations are performed assuming a diameter equal to the measured mean diameter of the starting material $(0.97 \mathrm{~nm})$. From our 

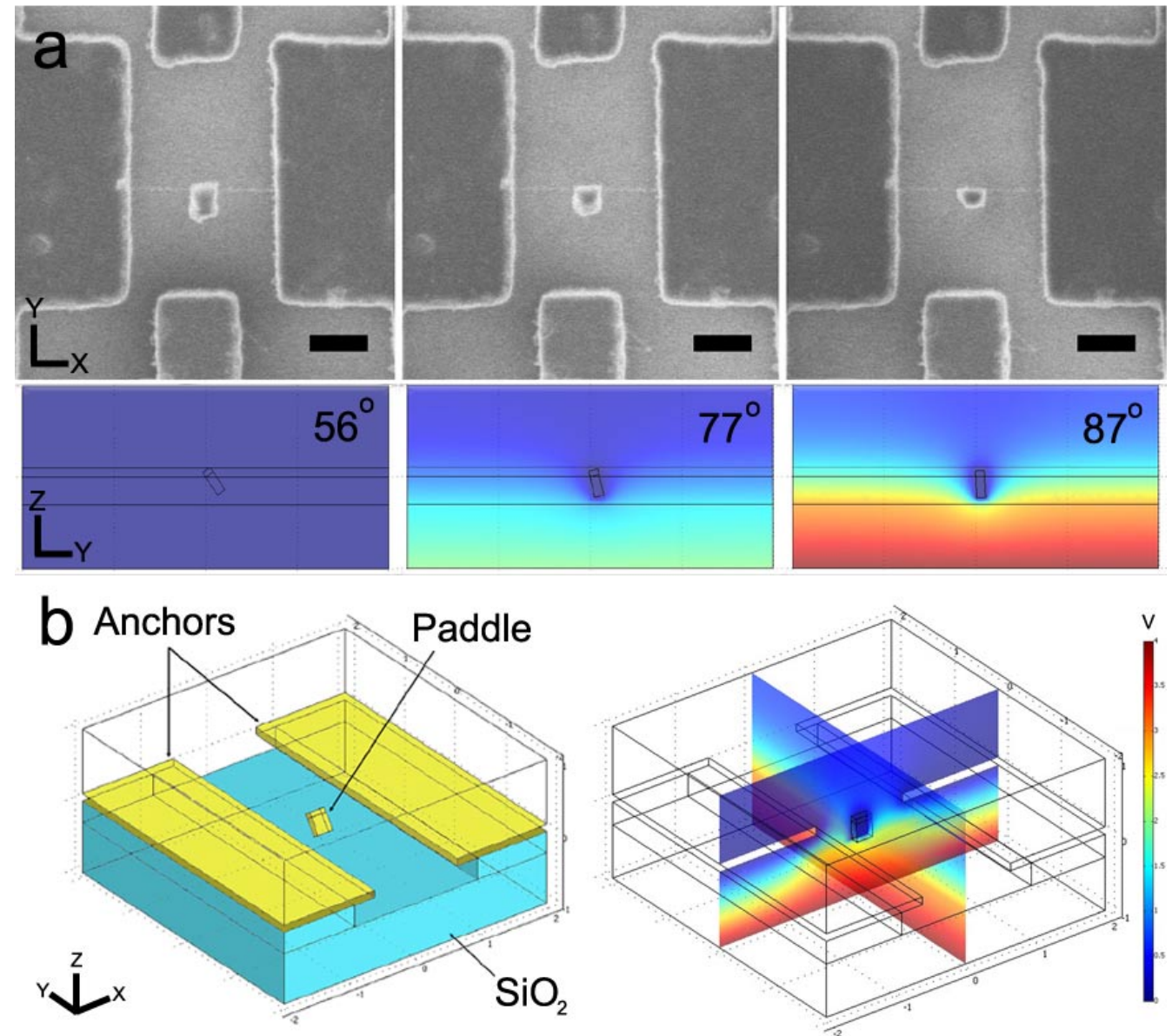

FIG. 2 (color online). (a) Sequential SEM images of device 6 at 0, 2, and 4 V applied voltage, respectively. Below each image is the FE calculated electric field potential (in the direction of the SWNT axis) of the device at each bias. (b) Labeled model of the device and overlaid color map of the FE calculated electric potential. Scale bars in (a) correspond to $500 \mathrm{~nm}$ and full color scale is $4 \mathrm{~V}$.

distribution, we expect $20 \%$ error, accounting for about $95 \%$ of all nanotubes on the surface. Differences in radii alone may be able to explain the statistical variation of shear modulus from one SWNT to the next. Other sources of error can be found in the dimensional measurements directly from the SEM images and shape variation of the paddle, each about $10 \%$. The latter of these is mainly due to the model's not accounting for rough edges on the metal. We suppose that the significantly lower moduli (of samples 3 and 5, for example) could be due to increased defect density in those specific SWNT, or to the diameters being slightly more than 1 standard deviation above the mean.

After the initial series of deflections of one device, the imaging beam was turned off and an oscillating signal was applied. This signal was of low frequency $(<1 \mathrm{~Hz})$ and of amplitude large enough to deflect the given device to nearly $90^{\circ}$. After several hundred cycles, the procedure to measure shear modulus was performed again. Despite the repeated deflections, the device showed no measurable change in reaction to applied field. This indicates that the hysteresis in the value of $G$ that was found in MWNT devices [5] is not present in similar systems built on SWNT and supports the theory that the stiffening may result from intershell interactions. Additional studies may be able to clarify this phenomenon.
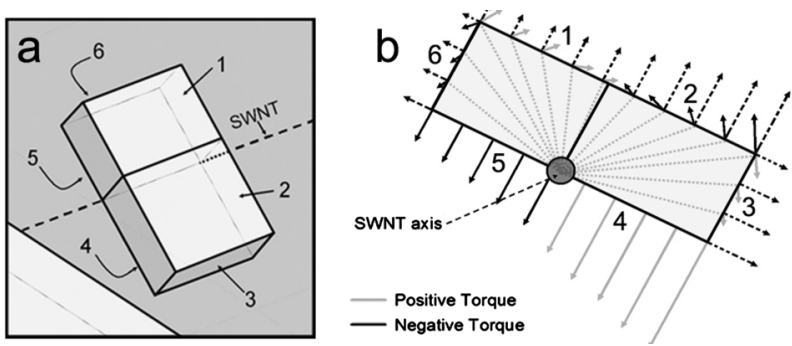

FIG. 3. (a) Details of the device model, showing the SWNT axis and the six surfaces of interest on the paddle. (b) Diagram of the electrostatic forces (dashed lines) acting on the paddle surfaces and the components of those forces that act as a torque. Moment arm of torques indicated with light gray lines drawn from SWNT axis. Figure drawn out of scale and at small deflection angle for clarity. 

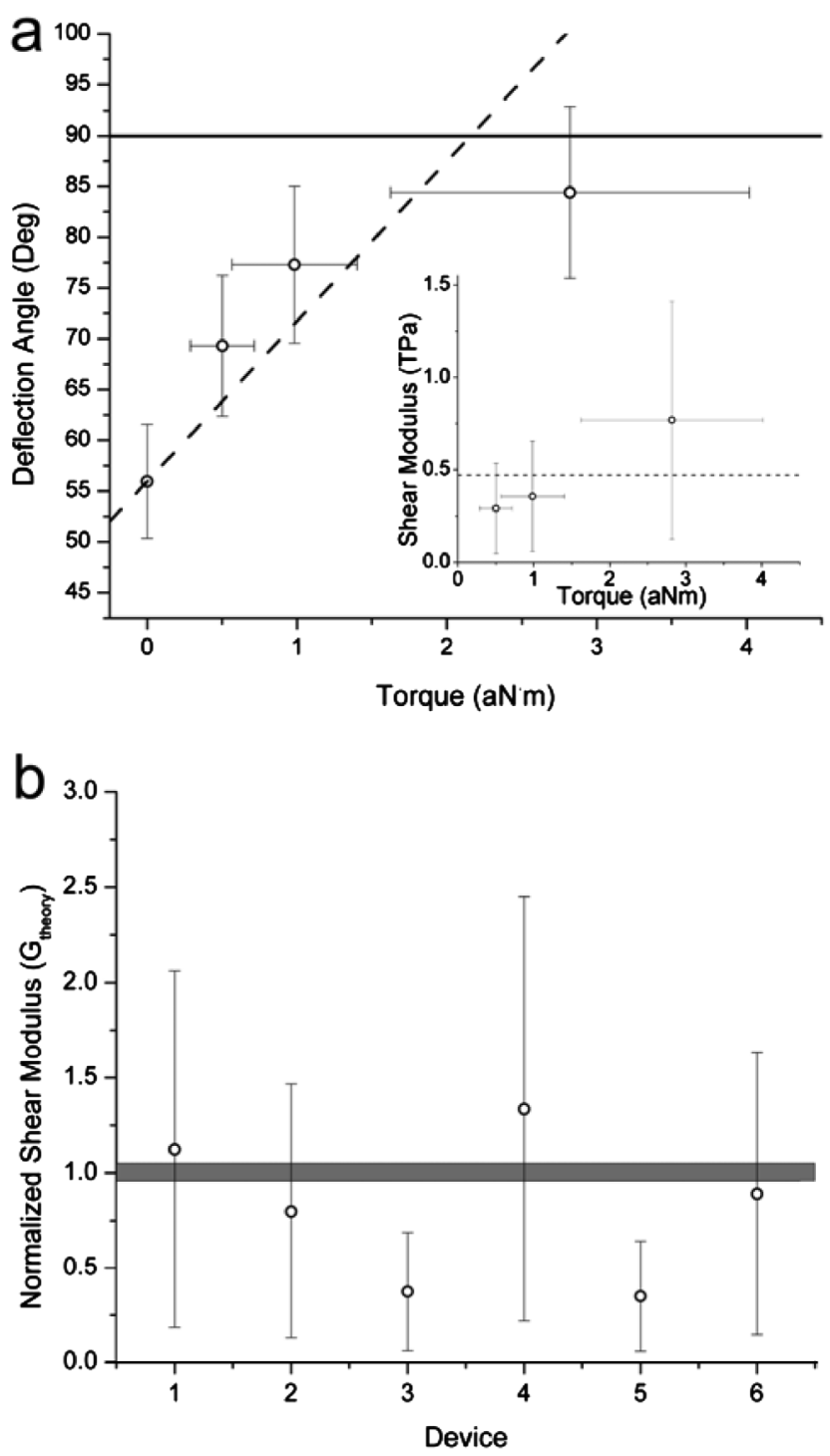

FIG. 4. (a) Deflection angle vs calculated torque for device 6 where $90^{\circ}$ is the maximum deflection attainable by our setup. Inset: measured shear modulus vs calculated torque for the same device. (b) Average shear modulus for each device, normalized to the average theoretical value $(0.455 \mathrm{TPa})$. The shaded region corresponds to the range of theoretical values over all chiralities in Ref. [14].

In conclusion, we have fabricated and characterized devices that incorporate an individual SWNT as a torsional spring. Application of torsional stress was accomplished with electrostatic forces, and material properties were calculated through modeling and FE analysis of the experimental data. Resultant shear moduli were found to agree with theoretical values. No change in shear modulus was observed with repeated device deflection. This methodology may be generalized to measure similar material properties of other nanowires and rods.

The authors thank NSF-ECS for funding this project. A.R.H. acknowledges the NASA GSRP program for financial support and R. Wincheski for helpful discussions.

[1] H. G. Craighead, Science 290, 1532 (2000).

[2] N. Yao, V. Lordi, S. X. C. Ma, E. Dujardin, A. Krishnan, M. M. J. Treacy, and T.W. Ebbesen, J. Mater. Res. 13, 2432 (1998).

[3] M.S. Dresselhaus, Science of Fullerenes and Carbon Nanotubes (Academic, New York, 1996).

[4] M. R. Falvo, G. J. Clary, R. M. Taylor II, V. Chi, F. P. Brooks, Jr., S. Washburn, and R. Superfine, Nature (London) 389, 582 (1997).

[5] P. A. Williams, S. J. Papadakis, A. M. Patel, M. R. Falvo, S. Washburn, and R. Superfine, Phys. Rev. Lett. 89, 255502 (2002).

[6] A. Fennimore, T. Yuzvinsky, W. Han, M. Fuhrer, J. Cumings, and A. Zettl, Nature (London) 424, 408 (2003).

[7] B. Bourlon, D. C. Glattli, C. Miko, L. Forro, and A. Bachtold, Nano Lett. 4, 709 (2004).

[8] S. J. Papadakis, A. R. Hall, P. A. Williams, L. Vicci, M. R. Falvo, R. Superfine, and S. Washburn, Phys. Rev. Lett. 93, 146101 (2004).

[9] J.C. Meyer, M. Paillet, and S. Roth, Science 309, 1539 (2005).

[10] P. A. Williams, S. J. Papadakis, A. M. Patel, M. R. Falvo, S. Washburn, and R. Superfine, Appl. Phys. Lett. 82, 805 (2003).

[11] Y. Li, J. Liu, Y. Q. Wang, and Z. L. Wang, Chem. Mater. 13, 1008 (2001).

[12] S. Gupta, K. Dharamvir, and V. K. Jindal, Phys. Rev. B 72, 165428 (2005).

[13] W.C. Young, Roark's Formulas for Stress and Strain (McGraw-Hill, New York, 1989), 6th ed.

[14] J. P. Lu, Phys. Rev. Lett. 79, 1297 (1997).

[15] B. I. Yakobson, C. J. Brabec, and J. Bernholc, Phys. Rev. Lett. 76, 2511 (1996). 